\title{
A CHAOTIC FUNCTION WITH SOME EXTREMAL PROPERTIES
}

\author{
J. SMITAL
}

ABSTRACT. For a continuous function chaotic in the sense of $\mathrm{Li}$ and Yorke the continuum hypothesis implies the existence of a scrambled set which has among others full outer Lebesgue measure.

A continuous function $f: I \rightarrow I$, where $I$ is a real interval, is called chaotic provided there is an uncountable set $S$ (a scrambled set) such that for each $x, y \in S$, $x \neq y$, and each periodic point $p$ of $f$,

$$
\limsup _{n \rightarrow \infty}\left|f^{n}(x)-f^{n}(y)\right|>0, \quad \limsup _{n \rightarrow \infty}\left|f^{n}(x)-f^{n}(p)\right|>0
$$

and

$$
\liminf _{n \rightarrow \infty}\left|f^{n}(x)-f^{n}(y)\right|=0
$$

where $f^{n}$ is the $n$th iterate of $f(\mathrm{cf}$. [1]). In the literature there is known no set $S$ with positive Lebesgue measure. On the other hand, it is easy to construct from a Cantor-type function a chaotic continuous function whose each scrambled set is a null set. In the present note we prove the following

THEOREM. Let $f:[0,1] \rightarrow[0,1]$ be defined by $f(x)=2 x$ for $x \leqslant \frac{1}{2}$, and $f(x)=2-$ $2 x$ otherwise. Then the continuum hypothesis implies the existence of a scrambled set $S$ for $f$ with outer Lebesgue measure 1 and such that the inequalities (1) can be replaced by

$$
\begin{aligned}
& \limsup _{n \rightarrow \infty}\left|f^{n}(x)-f^{n}(y)\right|=1, \\
& \limsup _{n \rightarrow \infty}\left|f^{n}(x)-f^{n}(p)\right| \geqslant \frac{1}{2} .
\end{aligned}
$$

First we prove some lemmas. In the sequel, $f$ is always the above-quoted function and $\mu$ is the Lebesgue measure.

LemMA 1. Let $I \subset(0,1)$ be an open interval and $M$ an infinite set of positive integers. Then there is $a G_{\delta}$ set $B=B(I, M) \subset(0,1)$ with $\mu(B)=1$ such that for each $x \in B$ there is infinitely many $n \in M$ with $f^{n}(x) \in I$.

Proof. Denote $f^{-n}(I)=\left\{x ; f^{n}(x) \in I\right\}$. For positive integers $m<n$ let $C_{m, n}=$ $\cup\left\{f^{-i}(I) ; i \in M, m<i<n\right\}$. First assume that there is a sequence $n(1)<n(2)<$ $\cdots$ of integers such that $\mu\left(C_{n(i), n(i+1)}\right)=1$ for each $i$; then clearly $B=$ $\bigcap_{i=1}^{\infty} C_{n(i), n(i+1)}$ is the desired set.

Received by the editors February 4, 1982.

1980 Mathematics Subject Classification. Primary 26A18; Secondary 54H20

(C) 1983 American Mathematical Society $0002-9939 / 82 / 0000-0661 / \$ 01.50$ 
Otherwise choose $n(1) \in M$ such that $\mu\left(C_{n(1), m}\right)<1$ for each $m>n(1)$, and put $B_{1}=f^{-n(1)}(I)$. Clearly $\mu\left(B_{1}\right)=\mu(I)=d$. Now assume by induction that $B_{i}$ are defined such that

$$
\text { each } B_{i} \text { is open and } B_{i}, \subset B_{i} \subsetneq(0,1) \text {. }
$$

$$
\begin{gathered}
\mu\left(B_{i} \backslash B_{i-1}\right)>\frac{d}{2}\left(1-\mu\left(B_{i-1}\right)\right), \\
f^{n(i)}(x) \in I \text { for each } x \in B_{i} \backslash B_{i-1},
\end{gathered}
$$

where $n(i) \in M, i=2,3, \ldots, k$. For $n>n(k)$ let $G_{n}$ be the system of intervals $\left(2^{-n} j, 2^{-n}(j+1)\right), j=0,1,2, \ldots$ which are contained in $(0,1) \backslash B_{k}$, and let $D_{n}=$ $\cup G_{n}$. Clearly $D_{n} \cap B_{k}=\varnothing$ and for some sufficiently large $n=n(k+1) \in M$ we have $\mu\left(D_{n}\right)>\frac{1}{2}\left(1-\mu\left(B_{k}\right)\right)$. Put $B_{k+1}=B_{k} \cup\left(f^{-n(k+1)}(I) \cap D_{n(k+1)}\right)$. Now it is easy to verify that (5) to (7) are satisfied for $i=k+1$. Denote $\lim _{h \rightarrow \infty} \mu\left(B_{h}\right)=b$. If $b<1$, then by (6) $\mu\left(B_{k+1} \backslash B_{k}\right)>d(1-b) / 2>0$ for each $k$, and consequently by (5), $\lim _{k \rightarrow \infty} \mu\left(B_{h}\right)=\infty$, a contradiction. Hence $b=1$.

Denote $E_{0}=\cup_{k=1}^{\infty} B_{k}$. Now if we repeat the construction starting from $n(1)$ satisfying the condition $n(1)>i$ we obtain an open set $E_{l}, i=1,2 \ldots$ with $\mu\left(E_{l}\right)=1$ and such that for each $x \in E_{i}$ there is some $n \in M, n>i$ such that $f^{n}(x) \in I$. It suffices to take $B=\bigcap_{1=0}^{\infty} E_{i}$ and the lemma is proved.

Lemma 2. There is a $G_{\delta}$ set $A \subset(0,1)$ with $\mu(A)=1$ such that for each $x \in A$.

$$
\liminf _{n \rightarrow \infty} f^{n !}(x)=0 \text { and } \limsup _{n \rightarrow \infty} f^{n !}(x)=1 \text {. }
$$

Proof. Put $M=\{n !\}_{n=1}^{\infty}, A_{k}=B((0,1 / k), M)$ and $A^{k}=B((1-1 / k, 1), M)$ (see Lemma 1). Then $A=\bigcap_{k=1}^{\infty}\left(A_{k} \cap A^{k}\right)$ has the desired properties.

Lemma 3. If $x \in A$ and $p$ is a periodic point of $f$, then (4) is true.

Proof. Let $k$ be the order of $p$. Then $f^{k n}(p)=p$ for each $n$, hence $\lim _{n \rightarrow \infty} f^{n !}(p)$ $=p$ and (4) follows from Lemma 2 .

Lemma 4. For each $x \in A$ there is a $G_{\delta}$ set $A(x) \subset A$ with $\mu(A(x))=1$ such that for any $y \in A(x)$, statements (2) and (3) are satisfied.

Proof. Let $M_{k}=\left\{m ; f^{m}(x)<1 / k\right\}$. Put $B_{k}=B\left((0,1 / k), M_{k}\right)$ and $B^{k}=$ $B\left((1-1 / k, 1), M_{k}\right)$. Now let $A(x)=A \cap \cap_{k=1}^{\infty}\left(B_{k} \cap B^{k}\right)$; by Lemma $1, A(x)$ has the desired properties.

Proof of Theorem. We use transfinite induction to construct the set $S$. By the continuum hypothesis there exists a well-ordering $\left\{P_{\alpha}\right\}_{\alpha<\Omega}$ of the system of perfect subsets $P$ of $(0,1)$ with $\mu(P)>0$, where $\Omega$ is the first uncountable ordinal. Let $x_{0} \in A \cap P_{0}$. When $\left\{x_{\alpha}\right\}_{\alpha<\beta}$ are defined such that for each $x=x_{\alpha}, y=x_{\gamma}$, where $\alpha<\gamma<\beta$, the inequalities (2) and (3) are satisfied, take $H=\bigcap_{\alpha<\beta} A\left(x_{\alpha}\right)$ (see Lemma 4). Then $\mu(H)=1$ since $H$ is a countable intersection of sets of full measure. Hence we can choose some $x_{\beta} \in H \cap P_{\beta}$. Denote $S=\left\{x_{\alpha}\right\}_{\alpha<\Omega}$. Clearly (2) and (3) are satisfied for any $x, y \in S, x \neq y$. Since $S \subset A$, Lemma 3 implies that for any $x \in S$ and any periodic point $p$ of $f$, it follows that (4) is true. And finally, since $S$ intersects each perfect subset of $(0,1)$ of positive measure, the set $S$ must have the full outer Lebesgue measure. Q.E.D. 
REMARK. The above quoted function $f$ has no scrambled set with a positive Lebesgue measure.

To see it assume the contrary: Let $A$ be a scrambled set for $f$ with $\mu(A)=\lambda>0$. Fix some integer $m$ such that $2^{m} \lambda>1$. Clearly $f^{m}$ maps each of the intervals $I_{i}=\left(2^{-m}(i-1), 2^{-m} i\right)$ linearly onto $(0,1), i=1, \ldots, 2^{m}$. Put $A_{i}=A \cap I_{i}$. Then $\Sigma_{i} \mu\left(f^{m}\left(A_{i}\right)\right)>1$ hence for some $i \neq j$ we have $f^{m}\left(A_{i}\right) \cap f^{m}\left(A_{j}\right) \neq \varnothing$. Consequently there are two different points $x \in A_{i}, y \in A_{j}$ with $f^{n}(x)=f^{n}(y)$ for every $n>m$, contrary to the first inequality in (1).

\section{REFERENCES}

1. T. Y. Li and J. A. Yorke, Period three implies chaos, Amer. Math. Monthly 82 (1975), 985-992.

Department of Mathematics, Komensky University, 842 is Bratislava, Czechoslovakia 